\title{
LASER ION SOURCE STUDIES AT CERN
}

John Tambini (reporting for the CERN laser ion source collaboration)

PS Division, European Organisation for Nuclear Research (CERN)

1211 Geneva 23, Switzerland

Submitted to S.P.I.E. Proceedings of the 4th International Workshop on Iodine Lasers and their Applications
Trest Castle, Czech Republic, 19-22 September 1995

\begin{abstract}
The plasma produced when a powerful laser pulse is focused onto a target surface in vacuum can provide a copious source of highly charged ions. Ions can then be extracted from the plasma to form a high current, short pulse length ion beam. Experimental laser ion sources have been the subject of investigation in medical physics and particle accelerator applications; a laser ion source is an option for the injection system of heavy ions for the Large Hadron Collider at CERN where a high intensity lead ion beam is required. This paper describes work carried out at CERN to develop a $\mathrm{CO}_{2}$ laser ion source.
\end{abstract}

\section{LASER PRODUCED PLASMA}

When a powerful laser pulse is focused onto a solid surface, the solid absorbs laser radiation, leading to localised heating and subsequent evaporation. Electrons in the cloud of vaporised material absorb laser radiation by inverse Bremsstrahlung and cause stepwise ionisation of metal atoms to form a plasma. After a short time of some nanoseconds, the plasma reaches a critical density whereupon absorption of laser radiation and thus plasma electron temperature increase dramatically, and the plasma becomes highly reflective to incoming laser light. The very hot dense plasma formed by this process undergoes a rapid expansion normal to the target surface, and a range of kinetic energy is imparted to the ions ${ }^{1}$.

The maximum ion charge state produced by this process is determined by the electron temperature of the plasma, since this sets the maximum ionisation energy achievable by plasma electrons. The controlling factor on the electron temperature is the power density of the laser focused onto the target surface. Figure 1 (a) shows the peak charge state produced from a lead target as a function of $\mathrm{CO}_{2}$ laser power density ${ }^{2}$. The plasma electron temperature is typically a few hundreds of electron volts in this regime. The distribution of charge states within the plasma can be measured using an energy selective electrostatic analyser to separate individual charge states in time, as illustrated in Figure 1 (b) for a tantalum target. This shows the maximum charge state produced is $23+$, with the peak of the distribution at $20+$. In a laser ion source the neutral plasma is allowed to drift a certain distance in a field-free region, before the ions are separated from the electrons and accelerated to form a beam by a negative voltage applied to an extraction system. Note that all charge states from the maximum down to 1+ are extracted, and ions within the beam possess an energy spread characteristic of the plasma in addition to the extraction energy. 
Fig. 1 (a): Lead ion peak charge state $\langle\mathrm{Z}\rangle$ as a function of $\mathrm{CO}_{2}$ laser power density.
1 (b): Time of flight spectrum for tantalum ions produced in $\mathrm{CO}_{2}$ laser plasma. 


\section{THE EXPERIMENTAL LASER ION SOURCE AT CERN}

\subsection{Target Chamber and beam extraction system}

A schematic diagram of the CERN laser ion source experimental set-up is shown in Figure 2. The laser beam enters the target chamber through a $\mathrm{NaCl}$ window and is focused back onto the target by a copper parabolic mirror with focal length $30 \mathrm{~cm}$. The target is in the form of a short rotatable cylinder and is generally lead or tantalum. Tantalum has a similar mass to lead and has the advantage of a much higher melting point, which results in less target material being sputtered onto the focusing mirror. The laser plasma passes through a hole in the centre of the mirror and further expands in a series of telescopic drift tubes held at the same high positive potential as the target chamber.

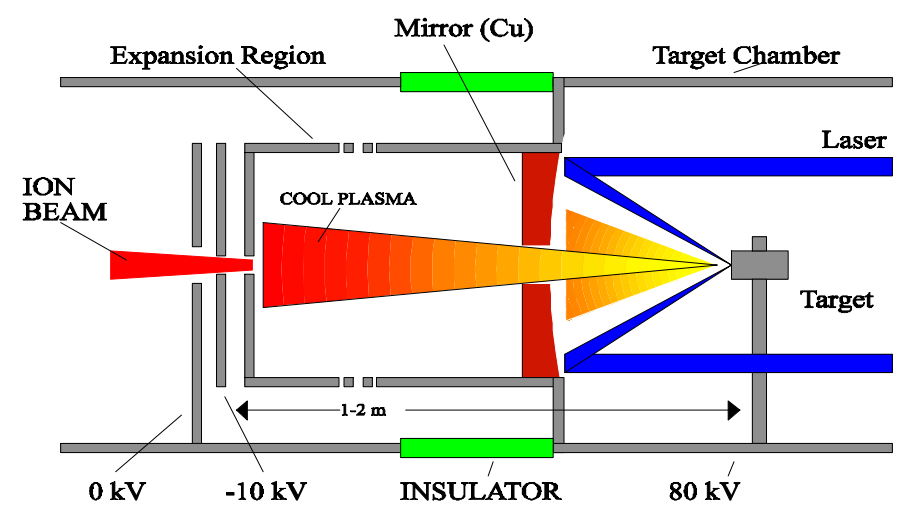

Fig. 2: Schematic layout of the CERN laser ion source experimental set-up

An ion beam is extracted from the plasma through successive electrode apertures held at $-10 \mathrm{kV}$ and ground potential respectively. The negative electrode is used to prevent secondary electrons released by ion beam striking the walls from re-entering the source. Both applied voltages are capacitively backed in order to remain at their nominal values during extraction of the ion beam current. A typical ion beam current pulse extracted from a tantalum target is shown in Figure 3, for a target to extraction drift length of $82 \mathrm{~cm}$, and extraction apertures of $30 \mathrm{~mm}$ diameter. The first part of the current pulse, from 3 to $8 \mu$ s is made up from high charge states, typically from $23+$ to $13+$; the later part of the pulse consists of the lower charge states. The peak current of the high charge state part of the pulse is $150 \mathrm{~mA}$. The extracted ion beam current can be controlled by the extraction system geometry - current increases directly with the extraction aperture area, and decreases as a function of the third power of extraction drift length. Also, the duration of the current pulse is directly proportional to the extraction drift length. Note that the ion beam is subject to a strong space charge force which causes the beam diameter to increase rapidly in the absence of any focusing fields.

Fig. 3: Ion beam current pulse extracted from a tantalum target

2.2 Laser

The CERN laser ion source study is based around a Lumonics TEA $601 \mathrm{CO}_{2}$ laser capable of supplying up to $50 \mathrm{~J}$ at a wavelength of $10.6 \mu \mathrm{m}$. The laser pulse consists of a fast initial spike (with FWHM $50 \mathrm{~ns}$ ) followed by a long "tail" 
lasting up to $1 \mu \mathrm{s}$, as shown in Figure 4 (a). Approximately half of the total laser energy is contained in the initial spike, with the other half in the tail of the pulse contributing only to low charge state production and evaporation of metal from the target. The laser pulse is delivered to the target via a $30 \mathrm{~m}$ path length to avoid parasitic oscillations from the reflective plasma, which would otherwise decrease the energy in the initial laser spike. The laser beam has a $10 \mathrm{~cm} x$ $\mathrm{cm}$ rectangular cross section, as shown in Figure 4 (b). This beam profile produces an irregular focal energy distribution rather than a spot, as shown in Figure 4 (c), resulting in a power density on the target of approximately $5 \mathrm{x} 10^{12} \mathrm{~W} / \mathrm{cm}^{2}$. The repetition rate of the laser is one shot per 20 seconds, governed by the re-charging time for the main discharge capacitors.

Fig. 4 (a): Output pulse from Lumonics TEA $601 \mathrm{CO}_{2}$ laser
4 (b): Laser beam profile

\author{
4 (c): Laser energy distribution \\ in focal plane
}

In an attempt to improve the distribution of energy in the focal plane, the laser resonator mirrors were changed from the original items with a rectangular profile to a set with a circular profile. This involves a trade-off between increasing the laser power density due to a better focal spot, and decreasing the laser energy due to utilising a smaller active volume of the laser cavity. Figure 5 (a) shows a comparison between the original and new beam profiles, and Figure 5 (b) shows part of a tantalum ion time of flight spectrum for original and new laser resonators. Figure 5 (b) shows that the maximum charge state achieved with the new resonator was $23+$, compared to $24+$ for the original. The laser energy was measured to be $60 \%$ lower for the new resonator, with the new resonator beam intensity shown in Figure 5 (b) also reduced by a similar factor. The disadvantage of lower ion beam yield with the new resonator is offset by the advantage of a smaller focal spot causing less sputtering onto the focusing mirror.

Fig. 5 (a): Original and new laser resonator beam profiles
5 (b): Tantalum ion time of flight spectra for original and new laser resonator

\subsection{Charge state distributions}

Information on the distribution of charge states within the plasma can be measured with a magnetic spectrometer. The unextracted plasma is allowed to drift a distance of $3.2 \mathrm{~m}$ to the entrance slit of the magnet. At a given magnetic field, only those ions with a certain momentum per unit charge are transmitted by the magnet. These ions are detected by a secondary electron multiplier which measures a time of flight spectrum for ions of different charge state. If a series of such measurements are taken at small steps across a range of magnetic fields, a composite plot of the charge state distribution can be obtained, as illustrated in Figure 6 (a) for a Tantalum target. The inherent energy spread in the 
plasma means that ions of each charge state describe an arc, with the highest energy arriving first and lower energy arriving later. The highest charge state $23+$ is seen at the lower left of the figure, with successively lower charge states down to $6+$ appearing towards longer arrival times. The short lines appearing at very early arrival times are due to light ion impurities such as oxygen and carbon.

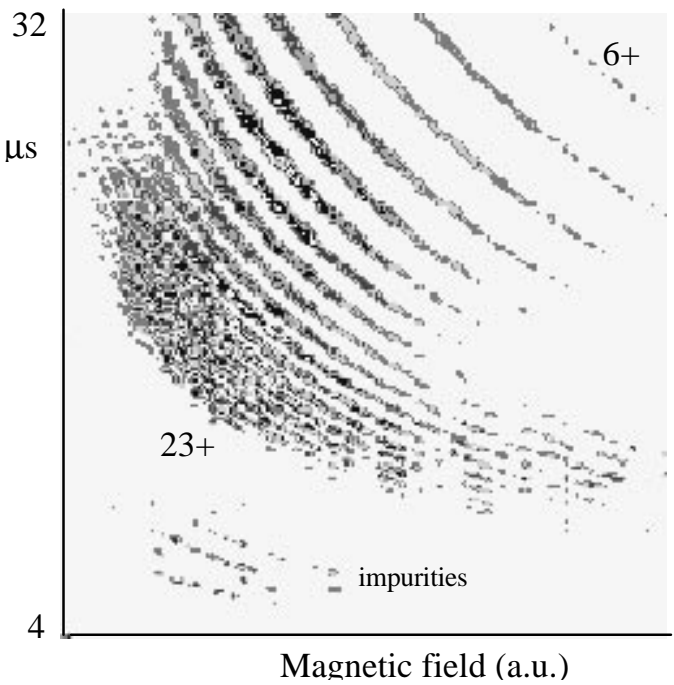

Fig. 6 (a): Charge state distribution for an unextracted tantalum plasma

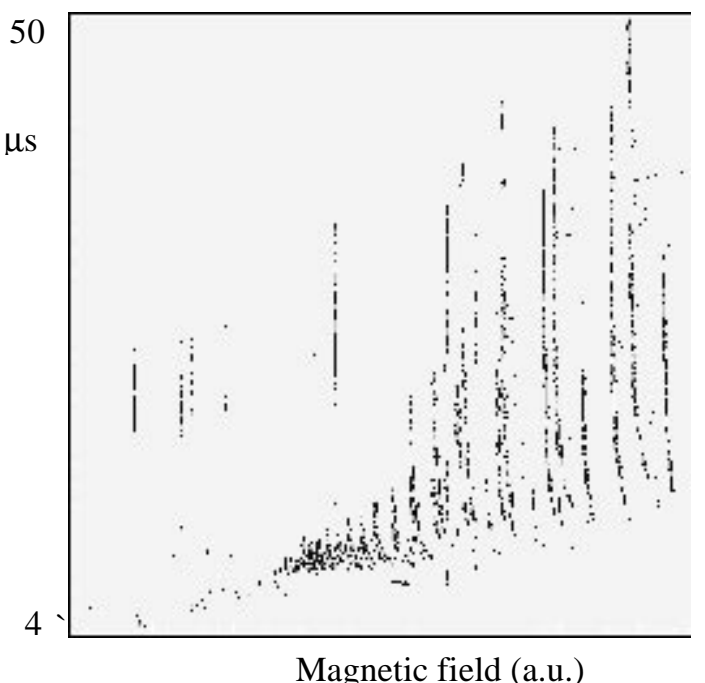

6 (b): Charge state distribution for an extracted tantalum ion beam

The same experiment can be repeated for an extracted ion beam, scanning over higher values of the spectrometer magnetic field. Figure 6 (b) illustrates such a scan for a $50 \mathrm{kV}$ extracted tantalum ion beam. In this case, each charge state appears as an almost vertical line since the inherent plasma energy spread is superimposed on the much higher energy gained from the extraction voltage. Again the high charge states appear at the lower left of the figure, and it can be seen that the early part of the extracted current pulse (compare with Figure 3) does consist of around 10 high charge states from $23+$ to $13+$.

\subsection{Beam emittance}

The transverse emittance of an ion beam is a measure of how the individual ion trajectories within the beam are distributed in phase space ${ }^{3}$. Beam emittance is an important parameter for the design of accelerating structures. The laser ion source beam emittance has been measured using the "pepper-pot" technique. A perforated metal foil, or pepperpot, is placed on the axis of the ion beam. The holes in the pepper-pot define a series of positions in phase space; ions pass through the holes and form an array of images on a micro-channel plate phosphor screen. The size of these images gives a measure of the spread in particle trajectory in the plane of the pepper-pot, and the angular position of images is calculated from the geometry of the system. The summation of this information from the whole beam allows one to plot a phase space ellipse and calculate the transverse beam emittance (the emittance equals the ellipse area divided by $\pi$ ).
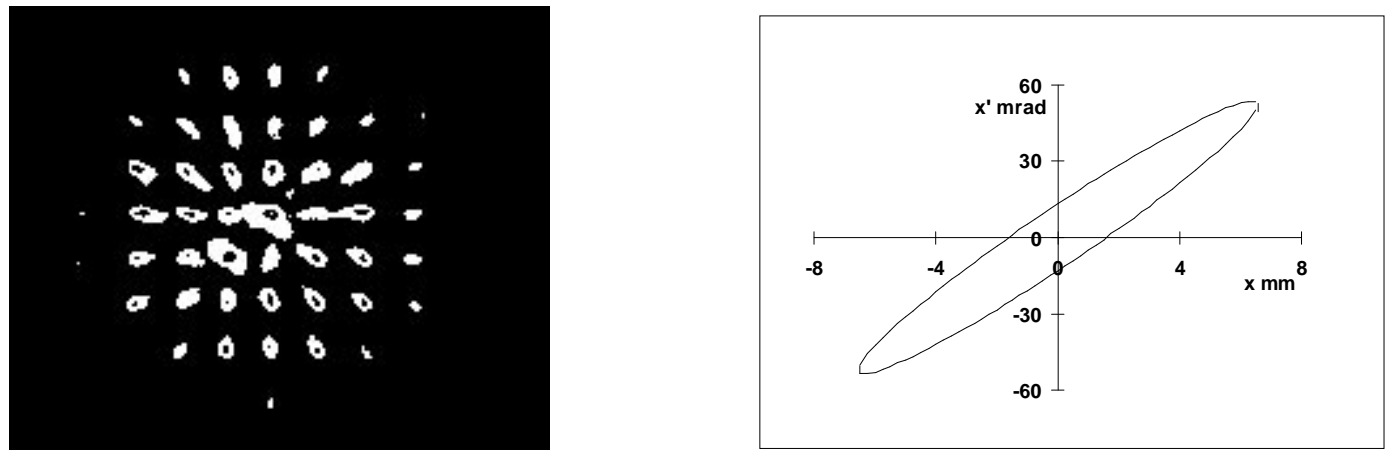
Fig. 7 (a): CCD camera image of pepper-pot

Fig. 7 (b): Phase space ellipse

Figure 7 (a) shows a CCD camera image of the pepper-pot array seen on the micro-channel plate phosphor screen for a tantalum ion beam. The pepper-pot consists of a $2 \mathrm{~mm}$ square array of $80 \mu \mathrm{m}$ diameter holes in a copper foil. The pepper-pot is mounted directly onto rear side of the ground extraction electrode, so that the ion beam is sampled before any appreciable space charge "blow-up" takes place. The extraction voltage was $66 \mathrm{kV}$, and extraction aperture diameter was $15 \mathrm{~mm}$. Figure 7 (b) shows a phase space ellipse calculated from this pepper-pot measurement, which yields a value for the transverse emittance of $105 \mathrm{~mm} . \mathrm{mrad}(5 \mathrm{rms})$. Note that the micro-channel plate was pulsed, with the measurement gate of $2 \mu$ s duration timed to coincide with the peak of the high charge state part of the current pulse. The emittance measured here thus includes information on several different charge states (from $23+$ to $13+$ ) which cannot be resolved.

\subsection{Energy spread reduction-modulated extraction voltage}

The rapid expansion of hot, dense plasma in a laser ion source results in a range of kinetic energy being imparted to ions. Normally, a DC extraction voltage is used to form the ion beam, so ions in the beam exhibit the same energy spread as the particles in the expanding plasma. In general, one is interested in accelerating only ions of a single charge state, so a low energy beam transport line (LEBT) is used to transport the chosen ions whilst also discarding the unwanted charge states. The spread in ion energies results in an undesirable increase in beam dimension introduced by the chromatic aberration associated with the focusing or filtering elements, and also makes acceleration by following RF structures more difficult. The inherent spread in ion beam energy can be reduced by modulating the extraction voltage in time, so that the higher energy ions leaving the source earlier see a lower extraction voltage than less energetic ions which emerge later. The extraction voltage modulation circuit is shown in Figure 8 (a). A positive DC voltage of $60 \mathrm{kV}$ is applied to the target chamber, and a negative voltage of $-7 \mathrm{kV}$ is stored in capacitor C. A thyratron trigger then discharges capacitor $\mathrm{C}$ to ground through resistance $\mathrm{R}$, which has the effect of superimposing a voltage ramp of $7 \mathrm{kV}$ with time constant $\tau=\mathrm{RC}$ onto the DC extraction voltage. Figure 8 (b) shows an oscilloscope trace of such a voltage $\begin{array}{lllllll}\text { ramp } & \text { with } & \mathrm{C} & 40 & \mathrm{nF} & \text { and }\end{array}$ $\mathrm{R}=140 \Omega(\tau=5.6 \mu \mathrm{s})$.

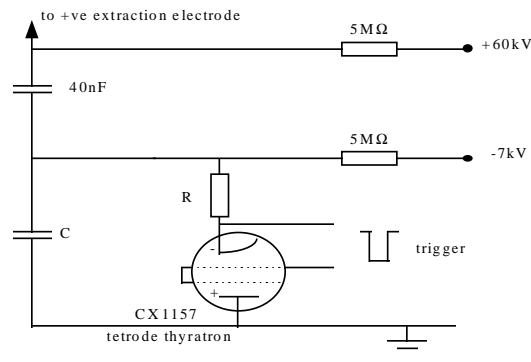

Fig. 8 (a): Extraction voltage ramping circuit

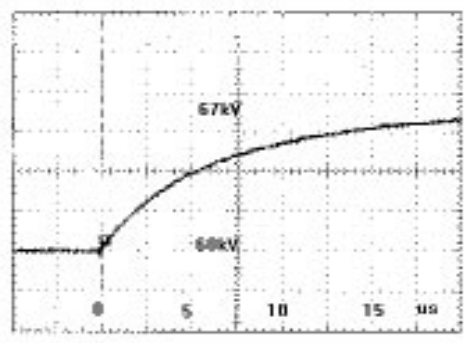

8 (b): Oscilloscope trace of extraction voltage ramp

The trigger delay, time constant $\tau$, and magnitude of the extraction voltage ramp are chosen to compensate the energy spread measured for the ion beam with DC extraction voltage. Figure 9 (a) shows a partial charge state distribution (measured as described in section 2.3) for tantalum ions of charge state $17+$ to $20+$, with a DC extraction voltage of 63 $\mathrm{kV}$. The range of magnetic field covered on the horizontal axis by each charge state corresponds to its spread in momentum, from which one can calculate the energy spread. In this case, the energy spread $\Delta \mathrm{E} / \mathrm{E}$ is about $11 \%$. Figure 9 (b) shows a corresponding partial charge state distribution for a modulated extraction voltage. The extraction voltage was modulated from $54.5 \mathrm{kV}$ to $64.5 \mathrm{kV}$, in a ramp triggered $1.6 \mu$ s after the laser pulse with time constant $\tau=5.6 \mu \mathrm{s}$; these conditions were chosen to overlap the voltage ramp with the position of the signal seen for DC extraction. It can be seen from Figure 9 (b) that each charge state extends over a much smaller range of magnetic fields on the horizontal axis, and the energy spread $\Delta \mathrm{E} / \mathrm{E}$ for this modulated extraction voltage case is reduced to about $2.5 \%$. 


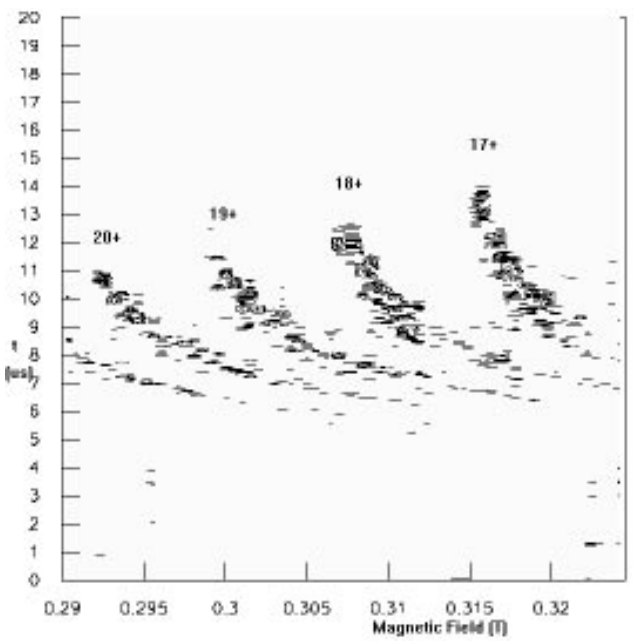

Fig. 9 (a): Tantalum $17+$ to $20+$ for DC extraction voltage. Energy spread $\Delta \mathrm{E} / \mathrm{E}=11 \%$

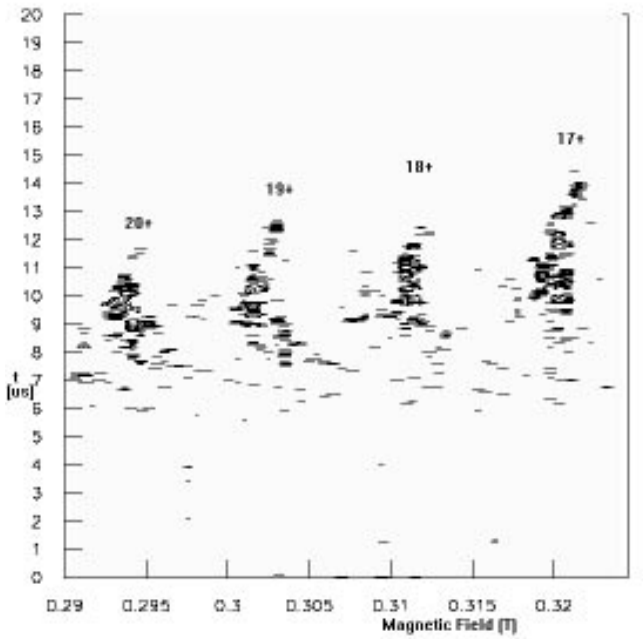

9 (b): Tantalum $17+$ to $20+$ for ramped extraction voltage. Energy spread $\Delta \mathrm{E} / \mathrm{E}=2.5 \%$

\subsection{Accelerated light ion beam}

Since the CERN accelerator system is concerned with heavy ions, all the work reported here so far has been involved with tantalum ions (as a practical experimental alternative to lead). However, a series of experiments was carried out with aluminium ions to make use of existing focusing elements and a radio frequency quadrupole (RFQ) accelerator which were designed for light ions (the RFQ was a contribution from the Lawrence Berkeley Laboratory to the oxygen ion accelerator project at CERN $)^{4}$. This work was done in order to prove the principle that a laser-produced ion beam could be contained and further accelerated. A schematic diagram of the experimental set-up is shown in Figure 10.

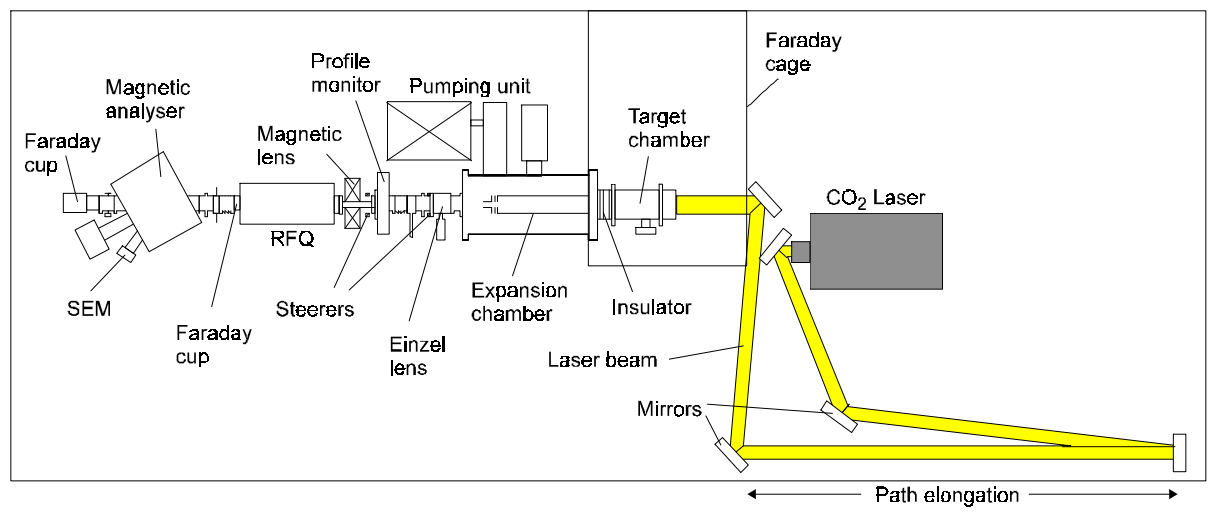

Fig. 10: Experimental set-up for aluminium ion beam acceleration.

Very high currents are produced from targets of light elements, so a long extraction drift distance of $2.2 \mathrm{~m}$ and small extraction apertures of $10 \mathrm{~mm}$ diameter were chosen to restrict the ion beam current. If too much current is extracted, there is a risk of breakdown between the extraction electrodes. The light ion RFQ was designed for particles with an input energy of $5.6 \mathrm{keV} / \mathrm{u}$ (i.e. energy per nucleon), which dictated an extraction voltage of $15 \mathrm{kV}$ for aluminium $10+$ ions. The LEBT consists of two elements; first an electrostatic Einzel lens to collimate the beam after extraction, and secondly a pulsed magnetic solenoid lens to focus the beam into the entrance of the RFQ. From a total current of $120 \mathrm{~mA}$ leaving the extraction system, the current at the entrance to the RFQ was measured to be $5 \mathrm{~mA}$, with the majority of the current loss being due to space charge blow-up of the beam before it enters the Einzel lens. The current after the RFQ was measured as $3 \mathrm{~mA}$. Analysis with the magnetic spectrometer showed that aluminium ions 9+ and 10+ had been successfully accelerated to $139 \mathrm{keV} / \mathrm{u}$. 


\section{LASER ION SOURCE DESIGN CRITERIA}

\subsection{Source requirements for the CERN Large Hadron Collider}

At present, lead ions are accelerated at CERN in the Super Proton Synchrotron (SPS) for fixed target experiments, using an Electron Cyclotron Resonance (ECR) ion source ${ }^{5}$. A schematic diagram of the CERN heavy ion accelerator sequence is shown in figure 11 . The ECR source produces $80 \mu \mathrm{A}$ of lead $27+$ ions in a long pulse of up to 1 ms duration. After acceleration in an RFQ and a linear accelerator (linac), this current pulse is multi-turn injected into the Proton Synchrotron Booster (PSB). Each of the four storage rings of the PSB is sequentially filled with 17 ion beam "buckets" of length $6 \mu \mathrm{s}$, where further acceleration takes place before injection into the Proton Synchrotron (PS). This process of multi-turn stacking is only $50 \%$ efficient, and combined with an inherent increase of beam emittance results in a substantial loss of beam luminosity. If the present ECR source were to be used to supply the Large Hadron Collider (LHC) then electron cooling and accumulation of the beam in the Low Energy Antiproton Ring (LEAR) would be required. This is the scheme considered in the conceptual design of the $\mathrm{LHC}^{6}$.

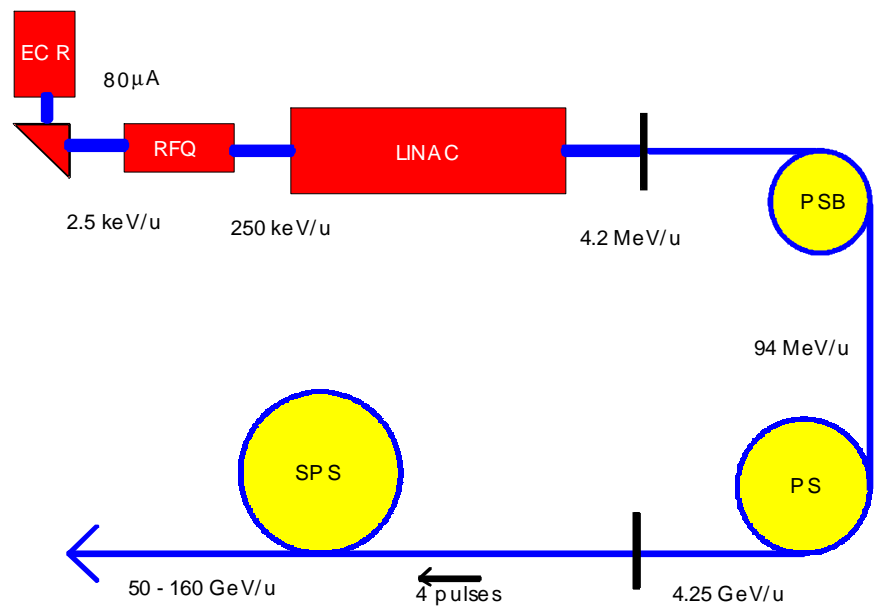

Fig. 11: The CERN heavy ion accelerator sequence.

A possible alternative to this scheme may be a laser ion source which could provide $6 \mathrm{~mA}$ of lead $25+$ in a pulse of duration $6 \mu \mathrm{s}$. This would allow single-turn injection, and would satisfy the luminosity requirements of the LHC. Further requirements for such a source would be $1 \mathrm{~Hz}$ repetition rate to give an LHC filling time of a few minutes, and a high number of shots $\left(10^{6}\right)$ without any intervention to allow un-interrupted runs over several weeks.

\subsection{Laser ion source design}

Based on results obtained with the present experimental ion source set-up, a laser ion source can be proposed to meet the above requirements of the LHC and associated injector sequence. The first, most fundamental requirement is the peak charge state of lead $25+$ which sets the laser power density required as $8 \times 10^{12} \mathrm{~W} / \mathrm{cm}^{2}$. The focal spot diameter chosen to achieve this power density is a trade-off between a small spot size which requires the lowest absolute laser energy, and a larger spot size which allows the use of a longer focal length mirror for greater flexibility in the design of the target illumination scheme. It is planned to use a mirror of focal length $2.6 \mathrm{~m}$, allowing the laser to be delivered to the target via a "folded beam" path, with the focusing mirror being protected from sputtered target material. This results in a focal spot diameter of $200 \mu \mathrm{m}$, which means the laser has to provide an $80 \mathrm{~J}$ pulse of $30 \mathrm{~ns}$ duration. The ion beam pulse duration of $6 \mu$ s required to match the PSB bucket timing sets the extraction drift length at $2.6 \mathrm{~m}$. The current required to satisfy the LHC luminosity is $6 \mathrm{~mA}$; to allow for some losses is transmission, an extraction aperture of $34 \mathrm{~mm}$ diameter is chosen which should provide a pulse of $10 \mathrm{~mA}$ of lead $25+$. Table 1 lists the important parameters of the proposed design. 


\begin{tabular}{|c|c|}
\hline peak charge state & lead $25+$ \\
\hline ion beam current $(25+)$ & $10 \mathrm{~mA}$ \\
\hline ion beam pulse duration & $6 \mu \mathrm{s}$ \\
\hline ion beam emittance (at $80 \mathrm{kV})$ & $100 \mathrm{~mm} . \mathrm{mrad}$ \\
\hline ion beam energy spread & $+/-2.5 \%$ \\
\hline laser power density & $8 \times 10^{12} \mathrm{~W} / \mathrm{cm}^{2}$ \\
\hline laser energy & $80 \mathrm{~J}$ \\
\hline laser pulse length & $30 \mathrm{~ns}$ \\
\hline focal spot diameter & $200 \mu \mathrm{m}$ \\
\hline extraction drift distance & $2.6 \mathrm{~m}$ \\
\hline extraction aperture diameter & $34 \mathrm{~mm}$ \\
\hline
\end{tabular}

Table 1: List of proposed laser ion source design parameters

The required laser energy is seen from Table 1 to be $80 \mathrm{~J}$. It was discussed in section 2.2 that a characteristic $\mathrm{CO}_{2}$ laser pulse consists of a fast initial spike followed by a long tail, with approximately half the total energy contained in each part. The energy contained in the tail of the pulse is effectively wasted, as it contributes only to low charge state production and evaporation. Further, if the laser has a highly non-Gaussian transverse beam profile, then more energy is lost in the far-field distribution in the focal plane. For these reasons, a master-oscillator / power amplifier laser design is proposed ${ }^{7}$. The master oscillator should include a low pressure tube to select single mode operation, and an electrooptical switch to prevent generation of the pulse tail. This clean single pulse would then pass through a telescope beam expander to the power amplifier. By using such a system, it is estimated that to provide $80 \mathrm{~J}$ of useful laser energy at the target, the total initial laser energy would need to be $100 \mathrm{~J}$.

Some practical points need to be taken into account in the design of the target chamber. With every laser shot, the expanding plasma strikes the inside walls of the target chamber and releases particles, causing a pressure rise. The requirement for $1 \mathrm{~Hz}$ operation places a high demand on the pumping capacity of the target chamber to minimise this effect. The design of the target itself is influenced by the requirement for up to 10 shots between interventions. Typically, 20 laser shots can be made on a single target position before the crater becomes too large and optimal focus is lost, leading to lower charge state yield. The diameter of this crater then determines how far the target has to be rotated to provide a new clean surface, which in turn dictates the total target area. In the present experimental set-up the laser pulse tail contributes greatly to the size of the crater, so measurements with the new tail-less laser pulse will be needed to finalise this design.

\section{CONCLUSION}

The work described here has shown that a laser ion source can provide a high current, short pulse heavy ion beam. Beam parameters such as emittance and energy spread have been measured and found to be acceptable in terms of beam transport and matching into further accelerator elements. A light ion beam has been successfully accelerated by an RFQ. A laser ion source design has been proposed which could meet the requirements of a lead ion source for the LHC. The next stage in the development of the CERN laser ion source is the construction of a dedicated LEBT and RFQ to demonstrate acceleration of a lead ion beam.

\section{ACKNOWLEDGMENTS}

This work has been carried out at CERN in close collaboration with the following institutes:

Institute for Theoretical and Experimental Physics (ITEP); Moscow, Russia

Troitsk Branch of the Kurchatov Institute (TRINITI); Troitsk, Russia

Institute of Physics, Academy of Science of the Czech Republic; Prague, Czech Republic

Institute of Plasma Physics and Laser Microfusion; Warsaw, Poland

Institute of Optoelectronics, University of Technology: Warsaw, Poland 


\section{REFERENCES}

1. I. G. Brown, The physics and technology of ion sources, Chapter 14, J Wiley, New York, 1989.

2. K. N. Makarov, Yu. A. Satov and A. P. Strel'tsov, Sov. J. Theor. and Exp. Phys, 106 (12), p. $1649,1994$.

3. J. Buon, "Beam phase space and emittance", CERN Accelerator School - Fifth General Accelerator Physics Course, Ed: S. Turner, Vol I, p. 89, CERN 94 - 01, Geneva 1994.

4. B. H. Wolf, K. Leible, P. Spadtke, N. Angert, J. Klabunde, B. Langenbeck, R. A. Gough, J. Staples, R. Caylor, D. Howard, R. MacGill, J. Tanabee, "Performance of the oxygen injector for the CERN Linac 1", GSI-86-2, GSI Darmstadt, 1986.

5. N. Angert, M. P. Bourgarel, E. Brouzet, R. Cappi, D. Dekkers, J. Evans, G. Gelato, H. Haseroth, C. E. Hill, G. Hutter, J. Knott, H. Kugler, A. Lombardi, H. Lustig, E. Malwitz, F. Nitsch, G. Parisi, A. Pisent, U. Raich, U. Ratzinger, L. Riccati, A. Schempp, K. Schindl, H. Schonauer, P. Tetu, H. H. Umstatter, M. Van Rooij, D. Warner, M. Weiss, "CERN heavy-ion facility design report" CERN 93-01, Ed: D. Warner, CERN Geneva, 1993.

6. LHC Study Group, "The Large Hadron Collider, conceptual design”, CERN AC 95-05 (LHC), Ed: P. Lefevre and T. Pettersson, CERN Geneva, 1995.

7. "Investigation of highly-charged heavy ion generation. $\mathrm{CO}_{2}$ laser ion source design.", Final report of annex to memorandum of understanding between the European Orgnaisation for Nuclear Research (CERN) and the Institute for Laser Physics (ILP), Troitsk, Moscow Region, 1995. 